\title{
The Application of Inertial Measurement Units and Wearable Sensors to Measure Selected Physiological Indicators in Archery
}

\author{
Zahari Taha', Rabiu Muazu Musa², Mohamad Razali Abdullah'2, Mohd Azrai Mohd Razman', \\ Chei Ming Lee', Faeiz Azizi Adnan ${ }^{3}$, Muhammad Amirul Abdullah' and Mainul Haque ${ }^{4 *}$ \\ 'Innovative Manufacturing Mechatronics and Sports Lab (iMAMs), Faculty of Manufacturing Engineering, \\ Universiti Malaysia Pahang, Pekan Campus 26600, Malaysia; \\ ${ }^{2}$ Faculty of Applied Social Sciences, Universiti Sultan Zainal Abidin, 21300, Terengganu, Malaysia; \\ ${ }^{3}$ Faculty of Mechanical Engineering, University Malaysia Pahang, Pekan Campus 26600, Malaysia; \\ ${ }^{4}$ Faculty of Medicine and Defense Health, National Defense University of Malaysia, Kem Sungai Besi, \\ 57000 Kuala Lumpur, Malaysia; runurono@gmail.com
}

\begin{abstract}
The requirement for objective techniques to observe physical action in its distinctive measurements has prompted the improvement and broad utilisation of motion sensors called Inertial Measurement Units (IMUs), which measures bodily movements. However, although these sensors have been utilised to measure postural balance in both clinical and some specific sports, little or no effort have been made to apply these sensors to the measurement of other physiological indicators in the sport of archery. This study aims to ascertain the postural balance, hand movement, muscular activation as well as heart rate of an archer. An archer was instructed to perform two balance standings, two hand movements and his muscular activations of flexor and extensor digitorum, as well as heart rate, were recorded using Shimmer sensors. The mean movement of $\mathrm{x}$ and $\mathrm{y}$-axis of the archer was used to correlate with the Pearson correlation for testing the validity of the sensors. Kolmogorov/Smirnov test was utilised to measure the reliability of the sensors over test re-test in two different tests. The coefficient of determination indicates some positive and negative significant relationships between some indicators. The Kolmogorov/Smirnov test re-test reveals a significant difference between all the indicators in both tests $A$ and $B, p<0.001$. The archer was able to present two types of postural standings and exhibited two hands movement while holding the bow. However, his heart rate demonstrated some variability during the executions of the movement in both tests. Thus, it could be concluded that the fusion sensors are reliable in measuring the aforementioned physiological indicators.
\end{abstract}

Keywords: Archery, Inertial measurements units, Movement analysis, Physiological indicators, Wearable sensors

\section{Introduction}

Current innovative advances reinforce the utilisation of Inertial Measurement Units (IMUs) and other wearable sensors as a practical alternative for the appraisal and measurement of exercise performance beyond the motion analysis laboratory ${ }^{1}$. These sensors offer various potential points of interest over conventional marker-based frameworks; they are miniature, cost-effective, simple to set-up and enable the appraisal of human movement in an unconstrained situation ${ }^{2}$. Fusion sensors are turning into undeniably well-known devices for evaluating and measuring human movement and other relevant parameters as they are available for synchronisation in numerous smartphones. This implies that these universal advancements may have the capacity to possibly quantify human movement and provide feedback with respect to the nature of the movements performed ${ }^{3}$. 
IMUs have been utilised in various ways from evaluating energy expenditure ${ }^{4}$, gait analysis ${ }^{5}$ and medical observations ${ }^{6}$. These sensors have additionally been utilised in the athletic field and sports such as skiing ${ }^{7}$ and golf $^{8}$. In a recent study, the usage of IMUs as a technique for tracking gym and rehabilitation exercises have been examined by Lin and Kulić. They assessed data collected from IMUs at the hip, knee and ankle during various lower limb works out. The information gathered from the IMUs was utilised to estimate joint angles; in which it was compared to those quantified via a marker-based motion capture analysis system. It was established from the investigation that the joint angles obtained through the IMUs were as accurate to those acquired by means of the aforementioned conventional methodology. Nonetheless, the quality of the activity performed was not quantified. In a different study, accelerometers were employed to survey exercise performance during gymbased resistance type exercises. The authors measured the movement quality in the context of exercise velocity performance ${ }^{3}$. However, diverse exercise objectives may require different movement speeds, and in that capacity, the appraisal of movement quality considering velocity alone does not offer an all-encompassing method for assessing exercise procedure. The purpose of the present study is to measure postural sway, hand movement, muscular activation as well as heart during execution of archery related techniques.

\section{Materials and Methods}

\subsection{Material's Description}

Shimmer wearable sensors are commercially available sensors developed by Shimmer Technology Company ${ }^{\mathrm{TM}}$. Shimmer's wearable sensor framework permits for direct and efficient capture, transmission, preparing and reflection of body detected information in real-time. The Shimmer body sensor platform and wearable detecting framework can be utilised in a variety of areas. As the equipment and apparatuses are considerably open and configurable, researchers can easily take the innovation and mould it to their own requirements. A screen capture of the shimmers used and the application is shown in Figure 1. The green colour indicates that the shimmer is connected while the amber colour shows that the shimmer is connecting.

\subsection{Experimental Protocol}

The experimental protocol in the present study was implemented in two parts. In the first part (Test A), the archer was instructed to sway from the centre of his gravity while holding the bow. Meanwhile, in the second part (Test B), the archer was permitted to limit the movement to enable the researcher to discover whether the sensors have the ability to differentiate the selected physiological indicators in the two types of the postural positions.

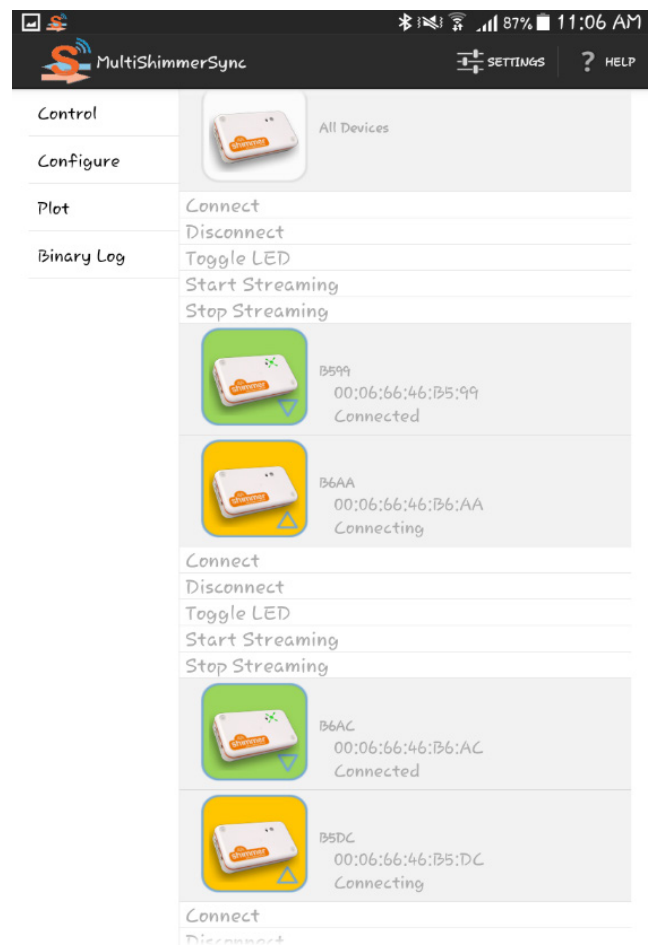

Figure 1. A screen capture of the shimmer sensors showing the main interface of the application.

\subsection{Data Collection Procedure}

A total of 4 Shimmer sensors were used in the present study to determine the postural balance, movement of the bow, muscular activations of the muscle flexor digitorum and extensor digitorum as well as the heart rate of the archer. To measure the postural sway, an accelerometer was firmly attached to the pelvic region of the archers as suggested by previous researchers to be the appropriate region when a centre of mass is to be determined ${ }^{10}$. To ascertain the movement of the bow, a shimmer sensor was attached to the hand of the archer holding the bow using a glove to hold the accelerometer tightly. However, bipolar electrodes were attached to the 
sensor by enabling the heart rate detector to determine the heartbeat of the archers from the wrist. Similarly, two shimmer sensors were strapped to the left muscle extensor digitorum and the right muscle flexor digitorum to obtain Electromyography (EMG) signals during the performances of the archery related movements described previously. All the data were transmitted via Bluetooth in real time at a sampling rate of $51.2 \mathrm{~Hz}$ using an Android phone for further analysis. The areas of all the sensors attachments in the body are shown in Figure 2.

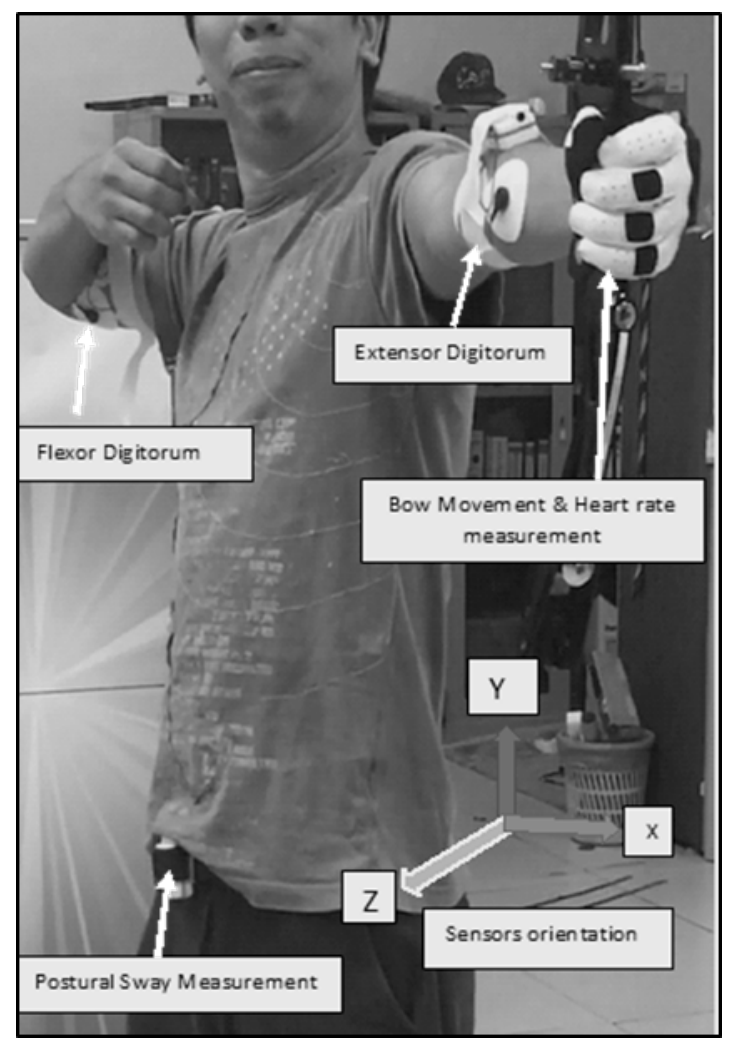

Figure 2. IMUs sensors' location attachments in the archer's body.

\subsection{Ethical Consideration}

Full information regarding the purpose of the research was explained to the archer, and a consent form was signed. All the procedures, protocol and apparatus for this study were endorsed by the Research Ethics Board of the Terengganu Sports Institute (ISNT) with a reference number 04-04/T-01/Jid 2.

\section{Data Analysis}

The calibrated mean movements of the archer from $\mathrm{x}$ and $y$-axis were used to correlate with the Pearson correlation coefficient for testing the validity of the sensors. On the other hand, the Kolmogorov/Smirnov test was applied to measure the reliability of the sensors utilised over test retest between tests $\mathrm{A}$ and $\mathrm{B}$ of all the movements measured at a confidence level of $p \leq 0.05$. Scatter plots were drawn to illustrate the summary for the relationship between the observed actions of the archer in the two different test measurements. The data for the total of five body actions were analysed and evaluated using MATLAB 2016a and XLSTART add in version 2014 USA for Windows.

\section{Results}

Table 1 tabulates the descriptive statistics of all the variables measured. The types of the observation (Tests $A \& B)$, the variables over which the athlete was examined, the minimum, maximum boundary, the mean as well as the standard deviation are displayed.

Table 1. Descriptive statistics of the Pearson correlation and the Kolmogorov/Smirnov test re-test on the measured variables

\begin{tabular}{lccccc}
\hline Variables & Obs. & Min. & Max. & M & SD \\
\hline Bow Mvmnt.T.A & 30 & -24.6 & 105.1 & 13.1 & 13.0 \\
Bow Mvmnt.T.B & 30 & -35.1 & 148.7 & 14.8 & 17.3 \\
Postural Sway. T.A & 30 & -2.2 & 0.5 & -0.5 & 0.3 \\
Postural Sway. T.B & 30 & -1.1 & 0.3 & -0.4 & 0.1 \\
Muscle Ex.Act.T.A & 30 & 0.0 & 0.0 & 0.0 & 0.0 \\
Muscle Ex.Act.T.B & 30 & 0.0 & 0.0 & 0.0 & 0.0 \\
Muscle Flx.Act.T.A & 30 & -0.1 & 0.1 & 0.0 & 0.0 \\
Muscle Flx.Act.T.B & 30 & -0.1 & 0.1 & 0.0 & 0.0 \\
Heart Rate.T.A & 30 & 88.0 & 105.0 & 95.3 & 4.4 \\
Heart Rate.T.B & 30 & 89.0 & 109.9 & 101.3 & 6.2 \\
\hline
\end{tabular}

Abbreviations: Bow Mvmnt.T. A = Bow Movement in test A, Bow Mvmnt.T.B = Bow Movement in test B, Postural Sway. T.A = Postural Sway in test A, Postural Sway. T.B= Postural Sway in test B, Muscle Ex.Act.T.A= Muscle Extensor Activation in test A, Muscle Ex.Act.T.B= Muscle Extensor Activation in test B, Muscle Flx.Act.T.A = Muscle Flexor Activation in test A, Muscle Flx.Act.T.B =Muscle Flexor Activation in test B, Heart Rate.T.A= Heart rate in test A, Heart Rate.T.B = Heart rate in test B.

Table 2 indicates the inferential statistics of the Kolmogorov/Smirnov test re-test. The two periods of testing, the observations, the D-statistics as well as the $\mathrm{p}$ values are shown. It can be detected from the table that tests 1 (observations $\mathrm{A}$ and $\mathrm{B}$ ) reveals a D-statistics of 0.24 and a corresponding $\mathrm{p}$-value of 0.0001 whereas test 2 (observations $\mathrm{A}$ and $\mathrm{B}$ ) indicates a D-statistics of 0.49 and a p-value of 0.0001 . However, test 3 (observations $\mathrm{A}$ and $\mathrm{B}$ ) reflects a D-statistics of 0.30 and a p-value of 
0.0001 while test 4 (observations $\mathrm{A}$ and $\mathrm{B}$ ) demonstrates a $\mathrm{D}$-statistics of 0.27 and a p-value of 0.0001 . Moreover, test 5 (observations A and B) exhibits a D-statistics of 0.47 and a $\mathrm{p}$-value of 0.0001 . The results from this table revealed that the p-value of all the tests $(1-5)$ is $<0.0001$ which explains that there is a statistically significant difference between tests $\mathrm{A}$ and $\mathrm{B}$ of all the measured actions. This confirmed the reliability of the sensors in evaluating as well as discriminating the two types of movement executed by the archer.

Table 2. Inferential Statistics of the Kolmogorov/ Smirnov test re-test

\begin{tabular}{|c|c|c|c|}
\hline Test-retest & Observation & $\mathrm{D}$ & P value \\
\hline \multirow[t]{2}{*}{ 1. Bow Movement. } & Test A & 0.24 & $0.0001^{\star}$ \\
\hline & Test B & & \\
\hline \multirow[t]{2}{*}{ 2. Postural Sway } & Test A & 0.49 & $0.0001^{*}$ \\
\hline & Test B & & \\
\hline \multirow[t]{2}{*}{ 3. Muscle Ex.Activation } & Test A & 0.30 & $0.0001^{*}$ \\
\hline & Test B & & \\
\hline \multirow[t]{2}{*}{ 4. Muscle Flx.Activation } & Test A & 0.27 & $0.0001^{\star}$ \\
\hline & Test B & & \\
\hline \multirow[t]{2}{*}{5 Heart Rate } & Test A & 0.47 & $0.0017^{\star}$ \\
\hline & Test B & & \\
\hline
\end{tabular}

${ }^{*}$ Significant at $\mathrm{p}<0.001$

Table 3 shows the Pearson correlation matrix of the variables observed. It can be seen from the table that heart rates in test A are significantly positively correlated with postural sway in test A which reveals that the more the archer moves, the higher the heart rate. However, a significant positive relationship was observed between heart rate in test $\mathrm{B}$ and bow movement in test $\mathrm{A}$ which indicates that the more the archer moves the bow, the greater the heart rate. In addition, a significant negative relationship was recorded between the heart rate in test $\mathrm{B}$ and the corresponding bow movement in test $\mathrm{B}$ which suggests that as the more the archer tried to keep the bow static, the lesser the heart rate. A significant positive association was also detected between the bow movement in test $\mathrm{A}$ and the corresponding postural sway in test $\mathrm{A}$ which means that the more the archer sways, the more the bow moves and vice-versa. Nevertheless, a significant negative relationship was recorded between the bow movement in test $\mathrm{B}$ and the postural sway in test $\mathrm{A}$ which proved that the more the archer tried to keep the bow static, the lesser the movement of the bow is observed. Conversely, a significant positive association was found between the bow movement in test B and muscle flexor activation in test $B$ which signifies that the more the archer attempted to keep the bow static, the greater the flexor muscles activation. Likewise, a negative association was observed between the archer's postural sway and the muscles flexor activation which indicates that the more the archer sways, the lesser is the flexor muscles activates and vice-versa.

Figure 3 displays the stability test conducted to determine the ability of the archer to maintain balance. The archer was instructed to stand and sway his centre of mass to ascertain whether the accelerometer used can detect any movement made by the archer while standing in test A (Blue colour). It can be observed from the figure that the archer has made some movements along the reference axis which indicates that the movement of the archer was not in a stable form. Moreover, the movement of the archer in test B (Red colour) is also evaluated. In test $\mathrm{B}$, the archer was instructed to maintain some level of stability while standing to allow the researchers to identify whether the balance of the archer can be detected by the

Table 3. Pearson correlation matrix among the variables

\begin{tabular}{lcccccccccc}
\hline Variables & $\mathbf{1}$ & $\mathbf{2}$ & $\mathbf{3}$ & $\mathbf{4}$ & $\mathbf{5}$ & $\mathbf{6}$ & $\mathbf{7}$ & $\mathbf{8}$ & $\mathbf{9}$ & $\mathbf{1 0}$ \\
\hline 1. Heart Rate.T.A & 1 & & & & & & & & & \\
2. Heart Rate.T.B & -0.16 & 1.00 & & & & & & & & \\
3. Bow Mvmnt.T.A & -0.11 & $0.84^{*}$ & 1.00 & & & & & & & \\
4. Bow Mvmnt.T.B & -0.39 & $-0.58^{*}$ & 0.08 & 1.00 & & & & & & \\
5. Postural Sway. T.A & $0.57^{*}$ & 0.25 & $0.54^{*}$ & $-0.74^{*}$ & 1.00 & & & & & \\
6. Postural Sway. T.B & -0.16 & 0.26 & 0.02 & -0.03 & 0.03 & 1.00 & & & & \\
7. Muscle Ex.Act.T.A & 0.26 & 0.07 & 0.06 & -0.04 & -0.06 & -0.03 & 1.00 & & & \\
8. Muscle Ex.Act.T.B & 0.24 & -0.13 & -0.01 & -0.18 & -0.16 & -0.08 & 0.03 & 1.00 & & \\
9. Muscle Flx.Act.T.A & -0.01 & -0.20 & -0.18 & -0.07 & -0.01 & -0.04 & 0.04 & -0.06 & 1.00 & \\
10. Muscle Flx.Act.T.B & -0.09 & 0.11 & 0.35 & $0.41^{*}$ & 0.29 & $-0.41^{*}$ & -0.20 & -0.22 & 0.03 & 1.00 \\
\hline *Significant at p<0.05 & & & & & & & & & &
\end{tabular}


accelerometer used. It can be seen from the figure that the pattern of the movement in test B was consistent which suggests that the movement of the archer is much more stable as compared to test $\mathrm{A}$. Based on this experiment, it can, therefore, be concluded that the sensor used is reliable to produce credible results over repeated measurement.

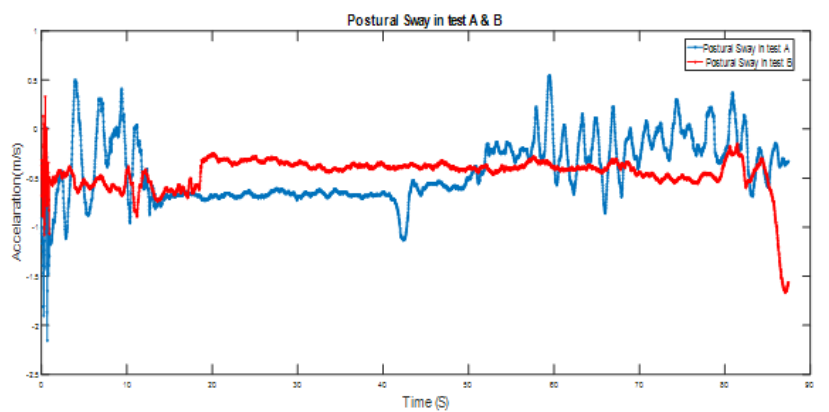

Figure 3. Comparative stability analysis between tests $\mathrm{A} \& \mathrm{~B}$.

\subsection{Measurement of the Hand Movement Using a Gyroscope}

Gyroscopes are sensors which evaluate the angular velocity of objects along the reference axes. Measuring physical activity utilising Gyroscope is favoured in light of the fact that angular velocity is proportional to external force and therefore can reflect the dynamics of human motion. Gyroscope information can be utilised to determine angular displacement data by integrating the angular velocity information with respect to time ${ }^{11}$.

Figure 4 reveals the comparative analysis of hand movement while holding the bow in test A (blue) \& B (red). In test $A$, the archer was instructed to the hold the bow with his hand and tries to move the bow to determine if the Gyroscope sensor is able to detect the movement of the hand while holding the bow. While in test B the archer was instructed to hold the bow, and tries to restrict his movement unlike in test A. It can be witnessed from the figure that there were some movements while holding the bow in both tests. However, the movement of the bow in test $\mathrm{B}$ appeared to be less compared to test $\mathrm{A}$ in which the movement is higher. Based on this result, it can be safely assumed that Gyroscope sensors can be used to measure the movement of the hand while holding the bow as suggested by the previous researchers ${ }^{12}$.

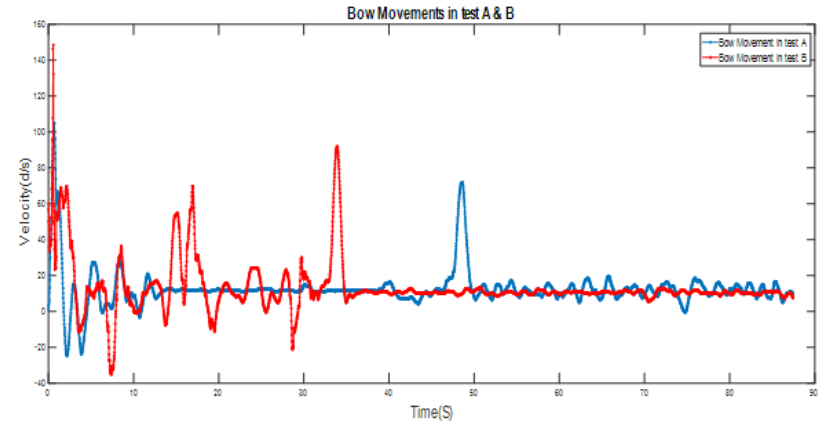

Figure 4. Comparative analysis of hand movement while holding the bow in test $\mathrm{A} \& \mathrm{~B}$.

\subsection{Measurement of Muscular Activation}

Figure 5 reveals the comparative analysis of extensor digitorum muscular activation in test $\mathrm{A} \& \mathrm{~B}$ while holding the bow. It can be observed that there was a higher muscular activation recorded from test B (red colour) in which the archer was instructed to maintain the bow in a static position. Therefore due to the higher demands placed on the muscles extensor in this test, the activation of the muscles appeared to be higher when compared to text A (blue colour) in which the archer was instructed to move the bow. This result alludes to the fact that the EMG sensors used could reliably measure all the activity performed by the working muscles during both aiming and releasing the arrow phases.

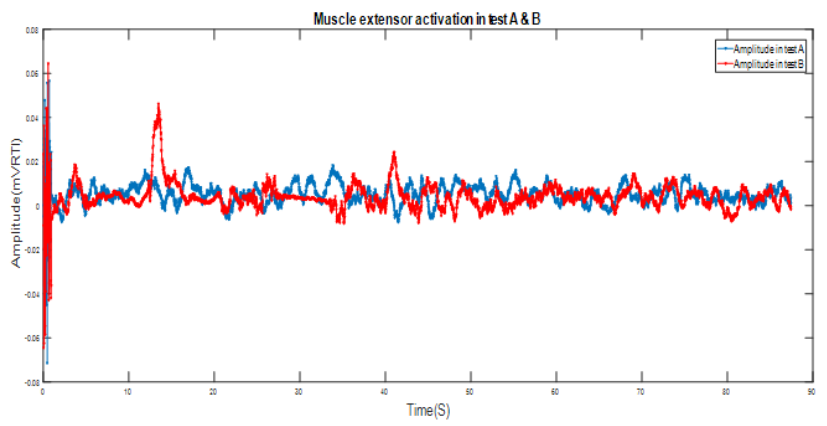

Figure 5. Comparative analysis of extensor digitorum muscular activation in test A\&B.

Figure 6 discloses the comparative analysis of flexor digitorum muscular activation in test $\mathrm{A} \& \mathrm{~B}$ while holding the bow. It can be observed that there was a higher muscular activation documented from test B (red colour) 
in which the archer was permitted to keep the bow static. This action has, therefore, placed a higher demand on the flexors muscles as compared to test $\mathrm{A}$ in which the archer was instructed to move the bow. This finding is consistent with the findings of previous researchers who observed that during the release of the arrow the flexor muscle digitorum played the major role and thus served as the highest activated muscle during the process ${ }^{13}$.

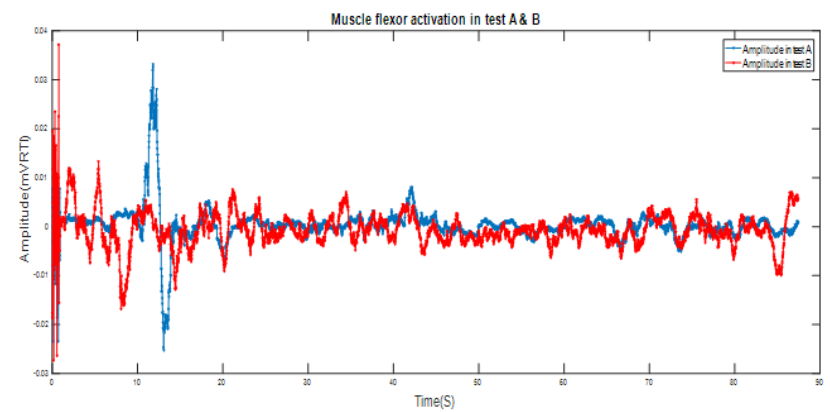

Figure 6. Comparative analysis of Flexor Digitorum muscular activation in test $\mathrm{A} \& \mathrm{~B}$.

\subsection{Measurement of Heart Rate}

Figure 7 illustrates a comparative analysis of the heart rate while holding the bow in test A \& B. From the figure, it can be observed that the heart rate recorded from the archer fluctuates. The heart rate in test B (red colour) is found to be higher when the archer was instructed to keep the bow static and to limit his movement unlike in test $\mathrm{A}$ where the archer was permitted to make some movement. The higher heart rate observed in test B can be attributed to the demand placed on the body to remain static while carrying the bow. The result reveals that the heart rate of the archer during the aiming and releasing stages of the arrow could be reliably recorded.



Figure 7. Comparative analysis of Heart rate while holding the bow in test $\mathrm{A} \& \mathrm{~B}$.

\section{Discussion}

The findings from the present study indicated that the fusion sensors employed have the potential to measure and discriminate some selected physiological indicators related to the sports of archery. The findings have further revealed that these sensors can be reliably depended upon to provide objective information of human performance.

The finding of the current study agrees with previous researchers who reported that IMUs had been utilised in a number of different applications ${ }^{4-6}$. Moreover, these sensors have additionally reported to be reliable in measuring activities related to skiing and golf ${ }^{7,8}$. The findings from the present study were found to be congruent with the study conducted by other researchers who assessed data collected from IMUs at the hip, knee and ankle during various lower limb workouts that were compared to readings obtained via the traditional motion capture (Mo-cap) analysis. The study concluded that it is without a doubt that the IMUs used were as accurate as the Mo-cap system. Similarly, the results of this study are in line with the previous authors who used accelerometers to survey exercise performance during gym-based resistance type exercises through the measurement of movement quality in the context of the velocity of exercise performance ${ }^{3}$. Nonetheless, it is worth to mention, that angular velocity values obtained from the accelerometers are preprocessed and are prone to drift errors in which may provide inaccurate results. Conversely, the employment of gyroscopes by means of IMU sensors would provide a more sound and credible results to be used for further analysis. In a nutshell, our study has discovered that the application of IMUs couple with additional wearable sensors i.e. heart rate and EMG sensors provide a more complete assessment of nontrivial physiological indicators that may discriminate the performance in archery.

Moreover, it was indicated in the present study that mobile devices (especially smartphones) have now become an essential part of daily life and with its constantly increasing processing power, they are more and more utilized for biomedical signal processing especially for ECG, EMG as well as the movement of activity analysis ${ }^{14,15}$. Therefore, it is apparent that the utilization of such technology may empower coaches through the data gathered to evaluate the performance of their athletes 
in various sport dimensions both quantitatively and qualitatively ${ }^{16}$.

\section{Conclusion}

The sport of archery is static in nature, for an athlete to achieve a maximum score in the sport he/she must be able to control their movement to hit a given target precisely. The current study has successfully evaluated postural sway, hand movement, muscular activation as well as heart rate attributed to the sport of archery in two distinct analyses. The sensors used in the study have demonstrated high sensitivity in the detection of any movement executed by the archer which is beneficial in analysing any form of movement during both aiming and releasing of the arrow. Furthermore, the study has indicated that inertial measurement units can be utilised to evaluate movements employed by the archers by providing information on every action executed which can go a long way in helping the archers to be aware of his/her movements and any incorrect techniques to help improve performance. In addition, the heart rate, as well as the EMG readings, may give a vital additional information about the physiological status of the archer during the shooting process.

\section{Acknowledgement}

The researchers wish to thank the National Sports Institute of Malaysia for providing the grant for the current study (ISNRG: 8/2014-12/2014).The researchers have no conflict of interest to declare.

\section{References}

1. Ahmadi A, Mitchell E, Destelle F, Gowing M, OConnor NE, Richter C, Moran K. Automatic activity classification and movement assessment during a sports training session using wearable inertial sensors. 2014 11th IEEE International Conference on Wearable and Implantable Body Sensor Networks; 2014 Jun. p. 98-103.

2. Giggins O, Kelly D, Caulfield B. Evaluating rehabilitation exercise performance using a single inertial measurement unit. Proceedings of the 7th International Conference on Pervasive Computing Technologies for Healthcare. Institute for Computer Sciences, Social-Informatics and Telecommunications Engineering; 2013 May; p. 49-56.
Available from: https://doi.org/10.4108/icst.pervasivehealth.2013.252061

3. Pernek I, Hummel KA, Kokol P. Exercise repetition detection for resistance training based on smartphones. Personal and Ubiquitous Computing. 2013; 17(4):771-82. Available from: https://doi.org/10.1007/s00779-012-0626-y

4. Rawson ES, Walsh TM. Estimation of resistance exercise energy expenditure using accelerometry. Med Sci Sports Exerc. 2010; 42(3):622-8. PMid:19952824. Available from: https://doi.org/10.1249/MSS.0b013e3181b64ef3

5. Kavanagh JJ, Menz HB. Accelerometry: A technique for quantifying movement patterns during walking. Gait Posture. 2008; 28(1):1-15. PMid:18178436. Available from: https://doi.org/10.1016/j.gaitpost.2007.10.010

6. Zhang M, Sawchuk AA. A customizable framework of body area sensor network for rehabilitation. 2009 2nd International Symposium on Applied Sciences in Biomedical and Communication Technologi; 2009 Nov. Available from: https://doi.org/10.1109/isabel.2009.5373703

7. Michahelles F, Schiele B. Sensing and monitoring professional skiers. IEEE Pervasive Computing. 2005; 4(3):40-5. Available from: https://doi.org/10.1109/MPRV.2005.66

8. Ghasemzadeh H, Loseu V, Jafari R. Wearable coach for sport training: A quantitative model to evaluate wrist-rotation in golf. Journal of Ambient Intelligence and Smart Environments. 2009; 1(2):173-84.

9. Lin JF, Kulić D. Human pose recovery using wireless inertial measurement units. Physiol Meas. 2012; 33(12):2099115. PMid:23174667. Available from: https://doi. org/10.1088/0967-3334/33/12/2099

10. Rocchi L, Chiari L, Cappello A, Horak FB. Identification of distinct characteristics of postural sway in Parkinson's disease: A feature selection procedure based on principal component analysis. Neurosci Lett. 2006; 394(2):140-5. PMid:16269212. Available from: https://doi.org/10.1016/j. neulet.2005.10.020

11. Chen KY,BassettDR. Thetechnologyofaccelerometry-based activity monitors: current and future. Med Sci Sports Exerc. 2005; 37(11 Suppl):S490-500. PMid:16294112. Available from: https://doi.org/10.1249/01.mss.0000185571.49104.82

12. Saunders JB, Inman VT, Eberhart HD. The major determinants in normal and pathological gait. J Bone Joint Surg Am. 1953; 35-A(3):543-58. PMid:13069544. Available from: https://doi.org/10.2106/00004623-195335030-00003

13. Altini M, Penders J, Roebbers H. An Android-based body area network gateway for mobile health applications. Wireless Health. 2010; 188-9.

14. Yi WJ, Jia W, Saniie J. Mobile sensor data collector using Android smartphone. IEEE 55th International Midwest Symposium on Circuits and Systems (MWSCAS); 2012 Aug. 956-9. Available from: https://doi.org/10.1109/mwscas.2012.6292180

15. Abdullah MR, Musa RM, Maliki ABHM, Kosni NA, Suppi- 
ah PK. Development of tablet application based notational analysis system and the establishment of its reliability in soccer. J Physical Edu Sport. 2016; 16(3):951-7.

16. Musa RM, Abdullah MR, Maliki AB, Kosni NA, Haque M. The application of principal components analysis to rec- ognize essential physical fitness components among youth development archers of terengganu, Malaysia. Indian Journal of Science and Technology. 2016; 11(9); 44-6. Available from: https://doi.org/10.17485/ijst/2016/v9i44/97045 